\title{
Anlotinib-A "Present" for Treatment of Spinal Epithelioid Hemangioendothelioma: A Case Report and Literature Review
}

kedong han

maoming people's hospital

zhijiang he

maoming people's hospital

jian wen

maoming peopl's hospital

yichao huang

maoming people's hospital

hua chen ( $\nabla$ chen1hua@163.com )

Maoming People's Hospital

\section{Case report}

Keywords: epithelioid hemangioendothelioma, spine, antiangiogenic, anlotinib, therapy, case report

Posted Date: January 5th, 2021

DOI: https://doi.org/10.21203/rs.3.rs-139107/v1

License: (9) This work is licensed under a Creative Commons Attribution 4.0 International License.

Read Full License 


\section{Abstract}

\section{Background:}

Epithelioid hemangioendothelioma (EHE) is a rare malignant vascular tumor with vascular and sarcomatous components that can affect the spine. There has been no standard second-line treatment for EHE with first-line chemotherapy.

\section{Case description:}

We report a 30-year-old male patient was admitted to our hospital on August 6, 2018.Complaining of waist and lumbar pain lasting for 1 month. Positron emission tomography-computed tomography (PETCT) showed osteolytic bone destruction and abnormal glucose metabolism and surgically removing the cancer. Post-operative diagnose was EHE. We gave a four-course chemotherapy combination with concurrent radiotherapy. About 1 month later, the patients whose cancers have progressed. A secondline treatment is given anlotinib to prevent the further spread of metastatic cancer.

\section{Conclusions:}

The presence of stable disease after take anlotinib lasted for up to six months and the OS was 16 months. Anlotinib explores therapy in EHE that recoup the defects and shortcomings in rare soft tissue sarcoma. We expect more patients can benefit from this treatment.

\section{Introduction}

Epithelioid hemangioendothelioma (EHE) is a rare vascular neoplasm that develops from vascular endothelial cells ${ }^{[1]}$. Compared with other bone tumors, spinal EHE is relatively rare, with only 52 cases reported to date. Although EHE can occur at any age, patients are usually between 20 and 30 years old. Age and gender may have no significant effect on morbidity ${ }^{[2]}$. EHE may present having single or multiorgan involvement. Bone lesions are undetected in $14 \%$ of the cases; while liver and/or lung show $51 \%$ of lesions in total, many other organs have been reported to be affected ${ }^{[2,3]}$. This disease is often misdiagnosed due to the lack of specific clinical symptoms and radiologic findings. Meanwhile, due to rather complicated pathophysiologic mechanisms and unclear etiopathogenesis, there are a few successful treatment options, which result in a poor prognosis in some cases ${ }^{[4]}$. Efficacy of anlotinib in advanced soft tissue sarcoma(STS) by age, gender and ECOG PS score(ALTER 0203)studies was presented in the oral report section at the American Society of Clinical Oncology (ASCO) annual meeting due to the excellent therapeutic efficacy of anlotinib in STS ${ }^{[5]}$. Herein, we report a case of spinal EHE patient treated with anlotinib after surgery and chemoradiotherapy. The treatment was able to prolong patient survival.

\section{Case Presentation}


In August 2018, a 30-year-old male patient was first referred to the hospital due to the main symptom of waist and lumbar pain for more than one month and really can't stand. He took celecoxib capsules for 1 week. The pain symptoms were not improved after painkiller. He had no medical history or medication. He did not have arthrodynia, rheumatoid arthritis (RA), ankylosing spondylitis (AS), and tuberculosis (TB) medical or family history, but had a 10-year history of smoking and alcohol abuse.Vital signs indicated temperature $37.0^{\circ} \mathrm{C}$; blood pressure of $110 / 73 \mathrm{mmHg}$; heart rate of 82 beats per minute; respiratory rate of 20 breaths per minute; and oxygen saturation of $98 \%$ in room air. Straight leg elevation test was positive.

Blood analysis of tumor markers, such as carcinoembryonic antigen (CEA), alpha fetoprotein (AFP), carbohydrate antigen 199 (CA199), total prostate-specific antigen (TPSA), and free prostate specific antigen (FPSA), was normal.

The MRI manifested as different kinds of bone destruction and soft-tissue tumor in spine. The two attempted to take tumor organization to make pathologic check both failed. We took a multi disciplinary team (MDT) for him and suggested him to order a PET-CT scan. PET-CT revealed tumors involving L3 vertebrae, soft tissue masses, stenosis of the spinal canal or the intervertebral foramen, and multiple lytic lesions. There was osteolytic bone destruction and abnormal accumulation of fluorodeoxyglucose (FDG) throughout L3 (Fig. 1C and D) and L5 (Fig. 2C and D).And experts say that for the patients, surgery may be a good alternative. The surgery included intracaps ule excision and marginal excision but the patients had unresectable mass.

On September 2, 2018 the postoperative pathology showed that epithelioid cells presented adenoid structure alignment and clear cytoplasm. Immunohistochemical staining was positive for CD31/ERG/Vimentin and TFE3 (++), $\beta$-catenin (++), and GFAP/CD34/P-CK/HMB45/ MelanA/SAM/Desmin (-) (Fig. 3).

The final diagnosis was EHE. doxorubicin aloneorin combination with other cytotoxic agents has been typically recommended as the first-line treatment in the past. Our decision was to use posterior vertebral tumor resection and spinal canal decompression, which were appropriate surgical treatments for severe osphyalgia. We gave a four-course chemotherapy with paclitaxel (TAX) plus Bevacizumab (PGB; CAPOX$B)$. This case can not adopt total-excision treatment, we can adopt radiotherapy after the operation (PTV: $5300 \mathrm{cGy} / 26 \mathrm{~F}$ ), The tumour progression for 1 months after the end of chemoradiotherapy. What does second-line treatment use? As yet Pazopanib is the only small molecule TKI approved by the FDA for second-line STS treatment to date, But there is no indication in China. Therefore angiogenesis inhibitor drug anlotinib was given (12 mg daily, days 1-14 of a 21-day cycle). Drug-related side effects, such as hand-foot skin reaction and hypertension, were observed. After 8 cycles of anlotinib treatment, PET-CT showed that there was no FDG accumulation throughout L3 (Fig. 1A and B) and L5 (Fig. 2A and B) vertebrae and soft tissue masses. There was no obvious progression of the tumor that revealed stable disease.The patient was discharged, and followed up on regular re-examination. PET-CT showed stable disease up to 6 months. The patient OS was 16 months after anlotinib treatment. 


\section{Discussion}

\section{Epidemiology and molecular mechanisms}

EHE is a rare malignant vascular tumor that can occur in any site of the body with low incidence $\left(1 / 1,000,000\right.$ per year). It accounts for less than $1 \%$ of all vascular tumors ${ }^{[6]}$. Soft tissue involvement is frequent, whereas spinal involvement is uncommon. EHE fatality rate was estimated to be $20 \%$, and it involved multiple bones with poor prognosis ${ }^{[2]}$. Molecular mechanisms are not fully known. Tsuneyosh was the first to report EHE of the bone in $1986^{[7]}$. In 1999, the first diagnostic imaging of EHE and detailed description suggested affection of young males having osteolytic lesions involving the cortex and cancellous bone of the lower extremities ${ }^{[8]}$. Although the pathogenesis of EHE remains unclear, molecular genetics studies represented a breakthrough in recent years, elucidating the etiology and the pathology. The putative malignant progression was paralleled by altered expression levels of tumor protein P53 (TP53), murine double minute2 (MDM-2), Caveolin-1 (CAV-1), and vascular endothelial growth factor (VEGF) ${ }^{[9]}$. Recent studies have shown that Bartonella spp induce the expression of vascular endothelial growth factor, thereby causing the proliferation of vascular endothelial cells ${ }^{[10]}$. The WWTR1 promoter encoding a specific fusion transcription factor participates in the development of tumorsWWTR1/CAMTA1 gene fusion by chromosome translocation $(1 ; 3)$ (p36. 3; q25) $)^{[11]}$. Studies have shown that FOSB (fused to ZNF36 or WWTR1) and FOS (with various fusion partners) were rearrangements accounting for $20 \%$ and $29 \%$ of the EHE cases, respectively ${ }^{[12]}$.

\section{Pathological Features}

The final diagnosis of EHE is established based on the pathological perspective, which is the "gold standard." CD34 and CD31 are recognized markers of endothelial cells. CD34 is non-specific and was estimated at $90 \%$ in malignant vascular tumors ${ }^{[13,14]}$. Although the expression of CD31 is limited to endothelial cells, macrophages, and platelets, it is a relatively specific vascular tumor marker ${ }^{[15]}$. Etsrelated gene (ERG) is a member of signal transducers and activators of the ETS transcription family, which is expressed by endothelial cells, and the ERG fusion gene can cause several types of tumors, such as prostate cancer, acute myeloid leukemia, and Ewing's sarcoma ${ }^{[16]}$. Miettinen et al. (2011) reported 43 cases of EHE, among which 42 expressed ERG and TFE3, one of the known fusion proteins with high expression ${ }^{[17]}$. Fli-1 is a nuclear transcription factor involved in cell proliferation and tumorigenesis, which can be used to distinguish benign vascular tumors from malignant vascular tumors ${ }^{[17]}$. Fli- 1 is more sensitive and specific than CD34 and CD31, and 38\% of all cases of EHE CK were positive ${ }^{[17,18]}$. EMA and smooth muscle antibody were negative in most cases or weakly focally positive in a few cases $^{[15]}$. One study showed that the expression positive rate of the WWTR1-CAMTA1 protein was $94 \%$ and that of YAP1-TFE was $6 \%$ in EHE ${ }^{[19]}$. Several studies have also shown the nuclear expression of FOSB protein in the subsets of epithelioid hemangioma (EH), including lesions showing angiolymphoid hyperplasia with eosinophilia pattern ${ }^{[1]}$. Additionally, EHE needs to be differentiated from diseases such 
as $\mathrm{EH}$, and epithelioid angiosarcoma (EA) should be differentiated from synovial sarcoma, malignant melanoma, and other malignant tumors with epithelioid morphology.

\section{Treatment Status}

We reviewed previous reports on spinal EHE and found no specific treatment plans or guidelines of prognostic value. Surgery for spinal EHE was the primary choice of treatment, and adjuvant chemotherapy was routine treatment ${ }^{[4]}$. Chemotherapy drugs to treat EHE including carboplatin, paclitaxel, carboplatin, etoposide, adriamycin, dacarbazine, and ifosfamide have been investigated ${ }^{[1,4]}$. Angiogensis plays an important role in tumor growth and metastasis, and anti-angiogenic therapy is a promising treatment of cancer. Overexpression of VEGF, VEGFR2, and VEGFR3 has been observed in pulmonary EHE samples ${ }^{[20]}$. Currently, bevacizumab combined with carboplatin and paclitaxel is the firstline treatment for EHE, achieving partial remission depending on patient's condition ${ }^{[4,20]}$. Disease stabilization can be achieved with anti-angiogenic agents, such as interferon, thalidomide, and celecoxib, showing to help people with EHE to live at least 6 months longer when given with bevacizumab combined with nab-paclitaxel ${ }^{[4]}$.Anlotinib is a new, small, orally administered multi-target tyrosine kinase inhibitor that strongly inhibits multiple targets, such as VEGFR, PDGFR, FGFR, and c-kit ${ }^{[21]}$. Anlotinib is in clinical trials for treatment of many human cancers, including non-small cell lung cancer (NSCLC), soft tissue sarcoma, gastric cancer, colorectal cancer, medullary thyroid cancer, differentiated thyroid cancer, and esophageal squamous cell carcinoma ${ }^{[5]}$.

Recent ALTER0203 study showed that Antinib significantly prolonged progression-free survival (PFS), improved objective response rate (ORR) and disease control rate (DCR), and showed good safety in advanced soft tissue sarcoma(STS).A multicenter, single-arm, phase II study subsequently explored anlotinib activity in patients with advanced STS who had failed previous conventional treatments and a phase IIB study to demonstrate the role of anlotinib in advanced STS ${ }^{[22]}$. Overall,233 patients who were treatment-in tolerant or progressed on anthracic line-based chemotherapy were enrolled. The ORR and DCR in the anlotinib group were significantly higher than those in the control group (ORR $10.13 \%$ vs $1.33 \%, P=0.0145 ;$ DCR $55.7 \%$ vs $22.67 \%, P<0.0001$ ). Additionally, anlotinb treatment significantly improved the median PFS relative to the control $(6.27$ months, $95 \% \mathrm{Cl} 4.30-8.40 \mathrm{vs} 1.47$ months, $95 \% \mathrm{Cl}$ $1.43-1.57, \mathrm{HR}=0.33, \mathrm{P}<0.0001)$. This trial further confirmed the efficacy and safety of anlotinib in advanced STS ${ }^{[23]}$.

At present, there are no specific treatment plans and guidelines of prognostic value for treatment of EHE [24].

Anlotinib is a novel multitarget receptor tyrosine kinase inhibitor that targets vascular endothelial growth factor receptor (VEGFR), fibroblast growth factor receptor (FGFR), platelet-derived growth factor receptors (PDGFR), and c-kit. It can prevent tumor angiogenesis and proliferative signaling ${ }^{[23]}$. ALTER0203 trial demonstrated that anlotinib significantly prolonged the median PFS in patients with advanced soft tissue 
sarcoma (STS)In this case, histopathological examination was performed to definitively diagnose EHE, a rare malignant vascular tumor with an aggressive clinical course, with a high tendency for both local recurrence and distant metastasis. We recommend treatment with anlotinib after surgery and postoperative adjuvant chemotherapy. The PFS was 6 months, and OS was 16 months, which were good results. Adverse reactions such as hand-foot skin reaction and hypertension were observed.

\section{Conclusion}

We report a spinal epithelioid hemangioendothelioma whereas the patients had unresectable mass at diagnosis. Anlotinib maintenance therapy after chemoradiotherapy as first-line for EHE significantly improves PFS and OS In this case report, anlotinib was shown to have good efficacy and safety for EHE. Large retrospective and prospective trials are needed to further confirm the efficacy and safety of anlotinib in the clinical treatment of EHE. Randomized clinical studies are required to further confirm the efficacy and safety of anlotinib in clinical treatment of EHE.

\section{Abbreviations}

EHE epithelioid hemangioendothelioma

MRI magnetic resonance imaging

PET-CT positron emission tomography-computed tomography

PFS progression-free survival

OS overall survival

RA rheumatoid arthritis

AS ankylosing spondylitis

TB tuberculosis

CEA carcinoembryonic antigen

AFP alpha fetoprotein

CA199 carbohydrate antigen 199

TPSA total prostate-specific antigen

FPSA free prostate specific antigen

FDG fluorodeoxyglucose 
TAX paclitaxel

PGB; CAPOX-B

MDT Bevacizumab

multi disciplinary team

TP53 tumor protein P53

MDM-2 murine double minute2

CAV-1 caveolin-1

VEGF vascular endothelial growth factor

ERG ets-related gene

EH epithelioid hemangioma

EA epithelioid angiosarcoma

NSCLC non-small cell lung cancer

\section{Declarations}

\section{Ethics approval and consent to participate}

The study was approved by the local ethics committee of Maoming People's Hospital, and written informed consent was obtained from the patients.

\section{Consent for publication}

Not applicable.

\section{Availability of data and materials}

The datasets used and/or analyzed during the current study are available from the corresponding author upon reasonable request.

\section{Competing interests}

The authors declare that they have no competing interests.

\section{Funding}

The work was funded by High-level Hospital Construction Research Project of Maoming People's Hospital. 


\section{Authors' contributions}

Hua Chen and Kedong Han analyzed, designed the study. Zhijiang He and Jian Wen work together to analyze data. Hua Chen wrote and edited the manuscript, and all authors read and approved the manuscript.

\section{Acknowledgments}

The authors would like to thank Dr. LeiGang Tian and Dr. Qin Liu from Maomin People's Hospital, Maomin, China, for their contributions to image processing; the High-level Hospital Construction Research Project of Maoming People's Hospital for financial support to conduct the research and preparation of the manuscript; and LetPub (www.letpub.com) for language assistance during the preparation of this manuscript.

\section{References}

1. Shon W, Billings SD. Epithelioid Vascular Tumors: A Review. Advances in anatomicpathology2019,26(3).

2. Inyang A, Mertens F, Puls F, Sumathi V, Inwards C, Folpe A, Lee CH, Zhang Y, Symmans P, Rubin B, Nielsen GP, Nguyen VH, Rosenberg AE. Primary Pseudomyogenic Hemangioendothelioma of Bone. The American journal of surgical pathology2016,40(5).

3. Schenker K, Blumer S, Jaramillo D, Treece AL, Bhatia A. Epithelioid hemangioma of bone: radiologic and magnetic resonance imaging characteristics with histopathological correlation. Pediatric radiology 2017, 47(12).

4. Rosenberg A, Agulnik M. Epithelioid Hemangioendothelioma: Update on Diagnosis and Treatment. Current treatment options in oncology 2018, 19(4): 19.

5. Chi Y, Fang Z, Hong X, Yao Y, Sun P, Wang G, Du F, Sun Y, Wu Q, Qu G, Wang S, Song J, Yu J, Lu Y, Zhu X, Niu X, He Z, Wang J, Yu H, Cai J. Safety and Efficacy of Anlotinib, a Multikinase Angiogenesis Inhibitor, in Patients with Refractory Metastatic Soft-Tissue Sarcoma. Clinical cancer research: an official journal of the American Association for Cancer Research 2018, 24(21).

6. Ko JS, Billings SD. Diagnostically Challenging Epithelioid Vascular Tumors. Surgical pathology clinics. 2015;8(3):331-51. doi:10.1016/j.path.2015.05.001.

7. Tsuneyoshi M, Dorfman HD, Bauer TW. Epithelioid hemangioendothelioma of bone. A clinicopathologic, ultrastructural, and immunohistochemical study. The American journal of surgical pathology 1986, 10(11).

8. Ellis TS, Schwartz A, Starr JK, Riedel CJ. Epithelioid hemangioendothelioma of the lumbar vertebral column: case report and review of literature. Neurosurgery 1996, 38(2).

9. Theurillat JP, Vavricka SR, Went P, Weishaupt D, Perren A, Leonard-Meier C, Bachli EB. Morphologic changes and altered gene expression in an epithelioid hemangioendothelioma during a ten-year course of disease. Pathology, research and practice 2003, 199(3). 
10. Mascarelli PE, Iredell JR, Maggi RG, Weinberg G, Breitschwerdt EB. Bartonella species bacteremia in two patients with epithelioid hemangioendothelioma. Journal of clinical microbiology 2011, 49(11).

11. Tanas MR, Sboner A, Oliveira AM, Erickson-Johnson MR, Hespelt J, Hanwright PJ, Flanagan J, Luo Y, Fenwick K, Natrajan R, Mitsopoulos C, Zvelebil M, Hoch BL, Weiss SW, Debiec-Rychter M, Sciot R, West RB, Lazar AJ, Ashworth A, Reis-Filho JS, Lord CJ, Gerstein MB, Rubin MA, Rubin BP. Identification of a disease-defining gene fusion in epithelioid hemangioendothelioma. Science translational medicine 2011, 3(98).

12. Cousin S, Le Loarer F, Crombé A, Karanian M, Minard-Colin V, Penel N. [Epithelioid hemangioendothelioma]. Bulletin du cancer 2019, 106(1).

13. Gong QX, Fan QH, Ding Y, Xiao QX, Wang QY, Tang JL, Song QY, Wang B. [Atypical epithelioid hemangioendothelioma: a clinicopathological analysis of eight cases]. Zhonghua bing li xue za zhi = Chinese journal of pathology 2019, 48(8).

14. Wu X, Li B, Zheng C, Hong T, He X. Clinical characteristics of epithelioid hemangioendothelioma: a single-center retrospective study. European journal of medical research 2019, 24(1).

15. Gill R, O'Donnell RJ, Horvai A. Utility of immunohistochemistry for endothelial markers in distinguishing epithelioid hemangioendothelioma from carcinoma metastatic to bone. Archives of pathology \& laboratory medicine 2009, 133(6).

16. Wang X, Qiao Y, Asangani IA, Ateeq B, Poliakov A, Cieślik M, Pitchiaya S, Chakravarthi B, Cao X, Jing X, Wang CX, Apel IJ, Wang R, Tien JC, Juckette KM, Yan W, Jiang H, Wang S, Varambally S, Chinnaiyan AM. Development of Peptidomimetic Inhibitors of the ERG Gene Fusion Product in Prostate Cancer. Cancer cell 2017, 31(6).

17. Miettinen M, Wang ZF, Paetau A, Tan SH, Dobi A, Srivastava S, Sesterhenn I. ERG transcription factor as an immunohistochemical marker for vascular endothelial tumors and prostatic carcinoma. The American journal of surgical pathology 2011, 35(3).

18. Perry KD, Al-Lbraheemi A, Rubin BP, Jen J, Ren H, Jang JS, Nair A, Davila J, Pambuccian S, Horvai A, Sukov W, Tazelaar HD, Folpe AL. Composite hemangioendothelioma with neuroendocrine marker expression: an aggressive variant. Modern pathology: an official journal of the United States and Canadian Academy of Pathology, Inc 2017, 30(11).

19. Flucke U, Vogels RJ, de Saint Aubain Somerhausen N, Creytens DH, Riedl RG, van Gorp JM, Milne AN, Huysentruyt CJ, Verdijk MA, van Asseldonk MM, Suurmeijer AJ, Bras J, Palmedo G, Groenen PJ, Mentzel T. Epithelioid Hemangioendothelioma: clinicopathologic, immunhistochemical, and molecular genetic analysis of 39 cases. Diagnostic pathology. 2014;9:131.

20. Park MS, Ravi V, Araujo DM. Inhibiting the VEGF-VEGFR pathway in angiosarcoma,epithelioidhemangioendothelioma,andhemangiopericytoma/solitary fibrous tumor. Current opinion in oncology 2010, 22(4).

21. Taurin S, Yang CH, Reyes M, Cho S, Coombs DM, Jarboe EA, Werner TL, Peterson CM, Janát-Amsbury MM. Endometrial Cancers Harboring Mutated Fibroblast Growth Factor Receptor 2 Protein Are Successfully Treated With a New Small Tyrosine Kinase Inhibitor in an Orthotopic Mouse Model. 
International journal of gynecological cancer: official journal of the International Gynecological Cancer Society 2018, 28(1).

22. Tian Z, Liu H, Zhang F, Li L, Du X, Li C, Yang J, Wang J. Retrospective review of the activity and safety of apatinib and anlotinib in patients with advanced osteosarcoma and soft tissue sarcoma. Investigational new drugs 2020, 38(5).

23. Wang ZM, Zhang SL, Yang H, Zhuang RY, Guo X, Tong HX, Zhang Y, Lu WQ, Zhou YH. Efficacy and safety of anlotinib, a multikinase angiogenesis inhibitor, in combination with epirubicin in preclinical models of soft tissue sarcoma. Cancer medicine 2020, 9(10).

24. Liu W, Jiang Q, Zhou Y. Advances of systemic treatment for adult soft-tissue sarcoma. Chinese clinical oncology2018,7(4).

\section{Figures}




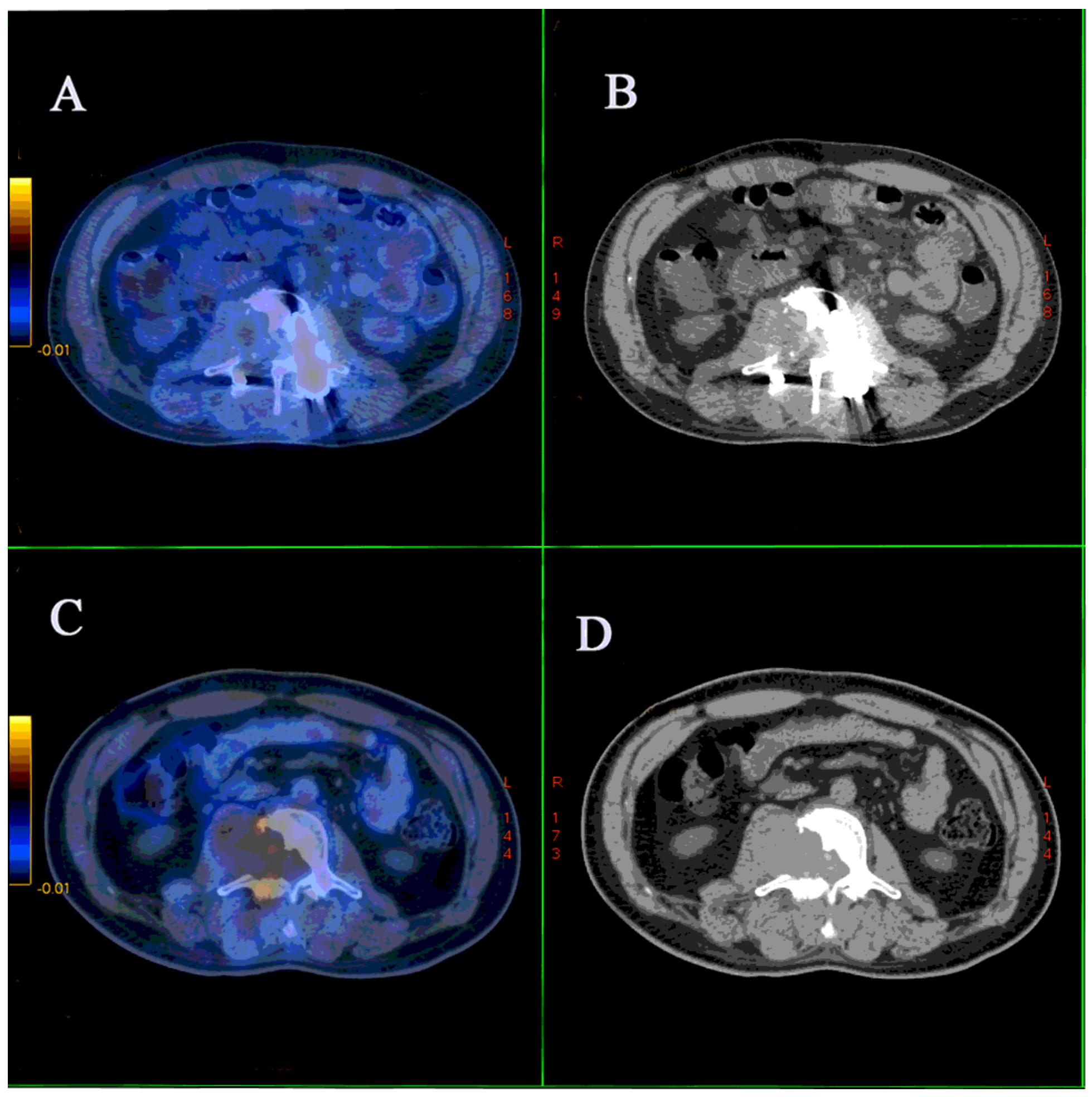

\section{Figure 1}

PET-CT showing the size of the tumor and accumulation of FDG at the L3 vertebrae prior-treatment figure( $C$ and $D$ ) and figure post-treatment ( $A$ and B). A: Normal accumulation of FDG of the L3 vertebrae after treatment. B: CT scan shows tumors tended to involve L3 vertebrae and soft tissue masses. After treatment, tumor did not progress compared with before therapy. C: Abnormal accumulation of FDG throughout the L3 vertebrae before therapy. D: CT scan shows tumors tended to involve L3 vertebrae and soft tissue masses. The sizes of tumors were $4.3 \times 5.8 \times 4.6 \mathrm{~cm}$ before therapy. 


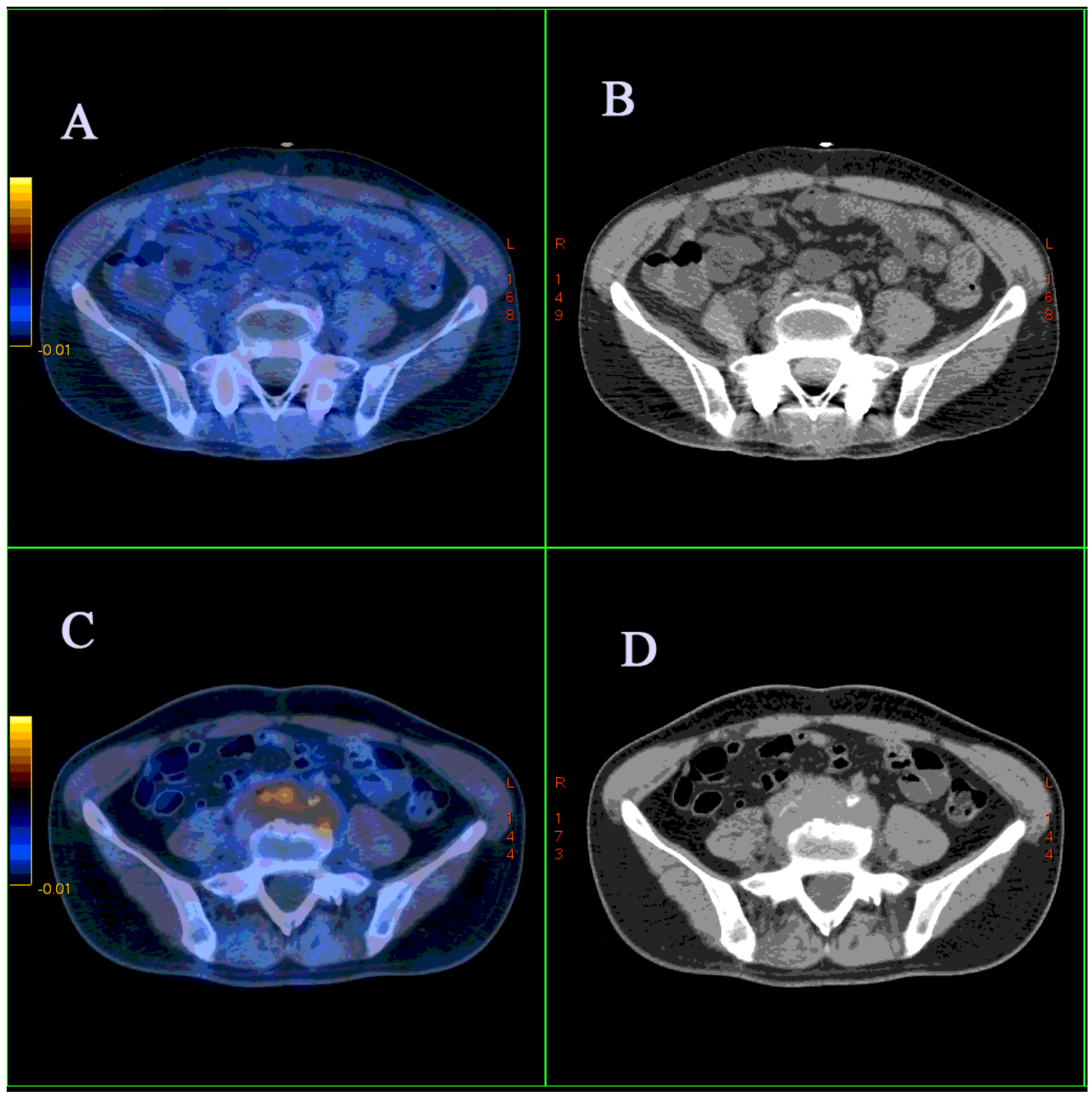

Figure 2

PET-CT showing the size of the tumor and accumulation of FDG at the L5 vertebrae prior-treatment figure( $C$ and $D)$ and figure post-treatment ( $A$ and $B$ ). A: Normal accumulation of FDG of the $L 5$ vertebrae after treatment. B: CT scan shows tumors tended to involve L5 vertebrae and soft tissue masses. After treatment, tumor did not progress compared with before therapy. C: Abnormal accumulation of FDG throughout the L5 vertebrae before therapy. D: CT scan shows tumors tended to involve L5 vertebrae and soft tissue masses. The sizes of tumors were $5.0 \times 6.4 \times 1.5 \mathrm{~cm}$ before therapy. 


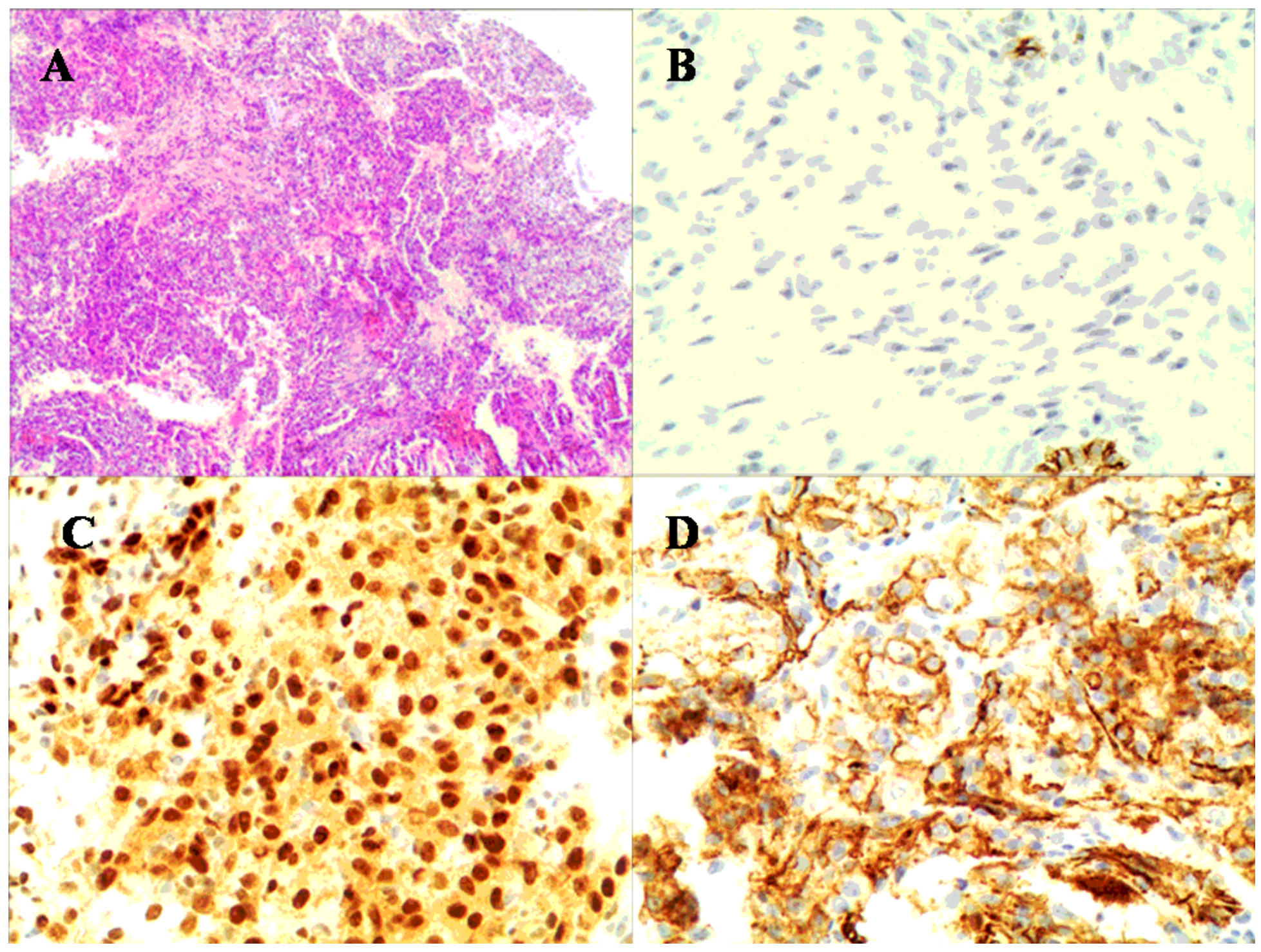

Figure 3

Hematoxylin and eosin and immunohistochemical stains. A: Epithelioid cells presented adenoid structure alignment and clear cytoplasm (HE, original $\times 200)$. B: Neoplastic cells stained negative for CD31 (original $\times 200$ ). C: Almost all cells were CD34 positive (original $\times 200$ ). D: Neoplastic cells stained positively for ERG (original $\times 200$ ). 